\title{
Conciliação de medicamentos em um hospital de ensino de Sergipe: lições aprendidas na implementação de um serviço
}

Medication reconciliation in a teaching hospital in Sergipe: lessons learned in implementing a service

\author{
P. A. Pimenta ${ }^{1}$; K. F. S. Santos ${ }^{1}$; C. M. L. Silva ${ }^{1}$; I. S. Passos²; I. M. C. Barros ${ }^{2}$; G. C. \\ Brito $^{2 *}$
}

${ }^{1}$ Residência Multiprofissional em Atenção Hospitalar à Saúde, Campus Professor Antônio Garcia Filho, Universidade Federal de Sergipe, 49400-000, Lagarto-Sergipe, Brasil

${ }^{2}$ Departamento de Farmácia, Campus Professor Antônio Garcia Filho, Universidade Federal de Sergipe, 49400-000, Lagarto-Sergipe, Brasil

*gisellecbrito@gmail.com

(Recebido em 07 de abril de 2020; aceito em 17 de agosto de 2020)

\begin{abstract}
Descrever a implementação da Conciliação de medicamentos em um Hospital de Ensino de Sergipe. Tratou-se de um estudo de ciência da implementação, que descreve a implementação da conciliação de medicamentos e os resultados preliminares deste serviço, no período de maio de 2016 a novembro de 2017. Inicialmente, foi realizado o diagnóstico situacional dos setores, seguido de fundamentação teórica, posteriormente foram elencados os indicadores a serem analisados: estrutura (estrutura física e recursos humanos), processos (fluxos de trabalho) e perfil dos pacientes atendidos (idade, sexo, perfil farmacoterapêutico). Assim, o próximo passo, foi descrever as etapas para implementação da conciliação de medicamentos. Além disso, caso outros setores do hospital almejem a implementação da conciliação de medicamentos, este estudo poderá auxiliar na projeção dos processos nas etapas de exploração, preparação e implementação inicial. Ademais, a promoção de atitudes colaborativas interprofissionais-contribui para maior aceitação das intervenções farmacêuticas, bem como, uma ampliação no número de farmacêuticos destinados a serviços clínicos auxiliará no atendimento da elevada demanda por serviços de conciliação.

Palavras-chave: Conciliação de medicamentos; Hospitais de Ensino; Ciência da Implementação.
\end{abstract}

To describe the implementation of the Medication Reconciliation in a Teaching Hospital of Sergipe. It was a study of implantation science, which describes the implantation of the Medication Reconciliation and the preliminary results of this service, from May 2016 to November 2017. Initially, a situational diagnosis of the sectors was carried out, followed by a rationale (physical structure and human resources), processes (workflows) and profile of patients attended (age, sex, pharmacotherapeutic profile). Thus, the next stage was to describe the steps for implementing Medication Reconciliation. In addition, if other sectors of the hospital aim to implement Medication Reconciliation, this study may assist in projecting the processes in the stages of exploration, preparation and initial implementation. The promotion of interprofessional collaborative attitudes contribute to greater acceptance of pharmaceutical interventions, as well as an increase in the number of pharmacists destined to clinical services will help to meet the high demand for conciliation services.

Key words: Medication Reconciliation; Teaching Hospitals; Implementation Science.

\section{INTRODUÇÃO}

Nas últimas décadas, tem-se observado um aumento significativo no uso de medicamentos dentro e fora do ambiente hospitalar, aumentando a probabilidade dos erros de medicação nos diferentes níveis de cuidado, expondo o paciente a um risco que poderia ser evitado [1,2]. Um erro de medicação pode ser definido como qualquer evento prevenível, que ocorre dentro do processo de utilização de medicamentos [3]. Em média, 10\% dos pacientes internados sofrem algum tipo de evento adverso e destes, $50 \%$ são evitáveis [4]. Dados como estes contribuíram para o conhecimento e conscientização sobre a importância dos erros de medicação e a utilização segura dos medicamentos se tornou um dos desafios mais importantes a serem enfrentados no contexto de cuidados de saúde [3].

Problemas farmacoterapêuticos podem ser minimizados através da conciliação de medicamentos, que é um processo formal de coleta de dados e de criação de uma lista apurada contendo todos os 
medicamentos em uso pelo paciente antes da admissão, transferência ou alta hospitalar conciliando as informações do prontuário, da prescrição, do paciente, de cuidadores, entre outras [5, 6]. A conciliação de medicamentos tem demonstrado ser uma das ferramentas mais eficazes para melhorar a segurança do paciente em transição de cuidados no ambiente hospitalar [7, 8].

Diversos estudos apontam para o sucesso clínico obtido pela conciliação [8, 9, 10]. Entretanto, estes estudos não possuem como foco descrever as etapas que viabilizaram a execução do serviço. Durante muitos anos a implementação dos serviços em diversas áreas, incluindo a área da saúde, ocorria de forma empírica, o que dificultava a identificação de fatores de sucesso ou falhas. Neste contexto, surge a ciência da implementação propondo o uso de teorias, modelos de processos e frameworks para obter insights nos mecanismos pelos quais a implementação é mais propensa a ter sucesso [11]. Diante do exposto, o presente estudo teve como objetivo descrever as etapas de implementação da Conciliação de medicamentos em um Hospital de Ensino de Sergipe, bem como elucidar as lições aprendidas em cada etapa.

\section{MATERIAIS E MÉTODOS}

\subsection{Desenho do Estudo}

Tratou-se de um estudo de ciência da implementação que descreve a implementação do serviço de conciliação de medicamentos e os resultados preliminares deste serviço realizado, no período de maio de 2016 a novembro de 2017, na Clínica Médica de um Hospital de Ensino de Sergipe.

As etapas de implementação do serviço de conciliação de medicamentos foram descritas, focandose na apresentação das fortalezas e limitações encontradas. Os dados foram analisados por meio de indicadores de estrutura (estrutura física e recursos humanos), processos (fluxos de trabalho) e resultados (clínicos) conforme a tríade avaliativa proposta por Donabedian (1988) [12]. Esta tríade avalia a qualidade dos serviços de saúde de forma que propicia um ciclo de qualidade, pressupondo que se há uma boa estrutura, o processo torna-se mais adequado e, consequentemente, garante bons resultados na assistência à saúde $[12,13]$.

Além disso, foi utilizado o Framework proposto por Moulin et al (2015) [14] que engloba as etapas dos processos de implementação para serviços clínicos providos por farmacêuticos: exploração, planejamento, início da operacionalização e implementação.

- Etapa 1: Exploração - tem como objetivo avaliar se a implementação é viável e criar condições para sua execução. O resultado desta etapa deve ser um plano de implementação claro para facilitar a instalação e implementação inicial do serviço.

- Etapa 2: Preparação - Tem como objetivo garantir que os farmacêuticos estejam preparados para se engajar na entrega do novo serviço e que os suportes estruturais e de nível de sistema estejam disponíveis para facilitar a implementação bem-sucedida.

- Etapa 3: Implementação inicial - visa integrar o novo serviço a rotina e depende do trabalho preparatório iniciado nos estágios de exploração e preparação.

- Etapa 4: Implementação total - O serviço é considerado sustentável e possui condições de ser avaliado integralmente. A implementação total de qualquer inovação pode levar de dois a quatro anos para atingir o impacto total. Deste modo, essa etapa não será apresentada neste estudo.

\subsection{Local do estudo}

O estudo foi realizado em um Hospital de Ensino do estado de Sergipe. O Hospital é administrado pela Empresa Brasileira de Serviços Hospitalares (EBSERH) e tem característica de porta aberta. É composto pelos seguintes setores: Pronto Atendimento, Clínica Médica, Clínica Cirúrgica, Centro Cirúrgico, Pediatria e Unidade de Terapia Intensiva. Realiza diferentes tipos de exames, como: eletrocardiogramas, radiografias, ultrassonografias e exames laboratoriais. O hospital conta com 155 leitos, entretanto, cerca de 78 leitos estão ativos. Atualmente a Clínica Médica possui 32 leitos ativos. O hospital dispõe de equipe multiprofissional na assistência, composta por médicos, psicólogos, nutricionistas, enfermeiros, fisioterapeutas, assistentes sociais, além de terapeutas ocupacionais e Residentes do Programa de Atenção Hospitalar à Saúde (farmacêuticos, enfermeiros, terapeutas ocupacionais, fonoaudiólogos, fisioterapeutas e nutricionistas). 


\subsection{Contexto do Estudo}

A implementação de serviços clínicos providos por farmacêuticos se iniciou com a chegada de duas farmacêuticas residentes da primeira turma de Residência Multiprofissional de Atenção Hospitalar à Saúde (2016). Quando a residência iniciou havia um quadro reduzido de farmacêuticos neste hospital, quatro profissionais, os quais desenvolviam apenas atividades logísticas. De acordo com a semana padrão da residência, cada residente ficava inserido em um dos setores do hospital durante três turnos na semana: segunda-feira à tarde, terça e quinta-feira pela manhã, com rotatividade de setor a cada três meses.

\subsection{Etapas para Implementação}

As etapas de implementação seguiram as recomendações de Moulin et al. (2015) [14], sob a ótica de Donabedian (1988) [12], como pode ser visto na Figura 1.

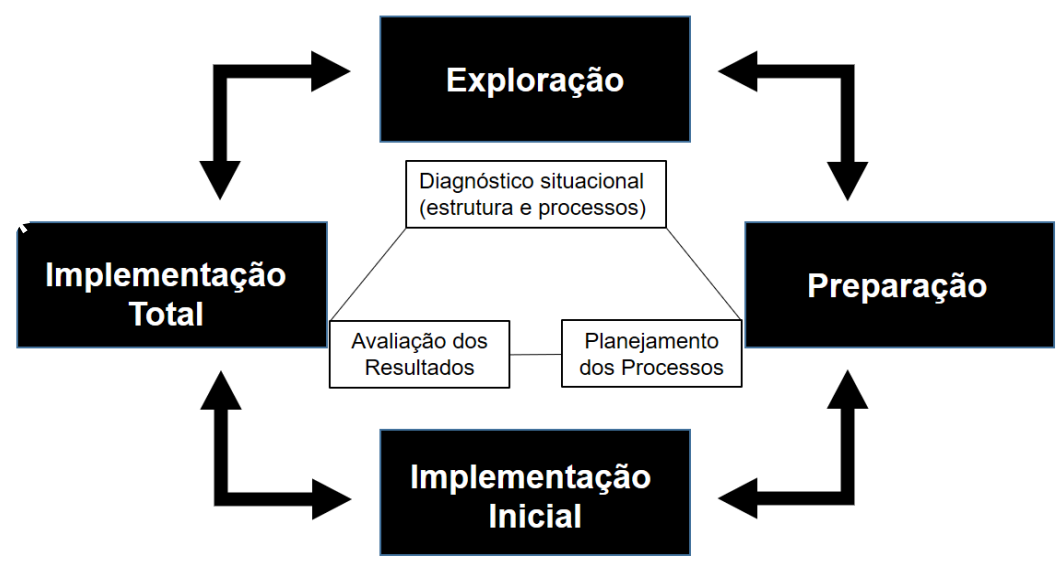

Figura 1: Fluxograma das etapas de implementação da Conciliação de Medicamentos em um Hospital de Ensino do Estado de Sergipe (2016-2017).

\subsubsection{Etapa 1 - Exploração}

Esta etapa ocorreu entre maio e junho de 2016 e contemplou o diagnóstico situacional do hospital por meio de observação participante mediada por um roteiro contendo os seguintes pontos: infraestrutura, recursos humanos, processos de trabalho e demandas, descritos a seguir.

- Infraestrutura: foram identificados setores que não necessitavam de amplas intervenções estruturais para a implantação de quaisquer serviços clínicos providos por farmacêuticos;

- Recursos humanos: as farmacêuticas residentes identificaram setores com profissionais predispostos ao trabalho multiprofissional-colaborativo e setores com baixa rotatividade profissional;

- Processos de trabalho: foram observados os setores que possuíam processo de trabalho bem definido, propícios a implantação de protocolos clínicos (monitoramento e avaliação do cuidado prestado), bem como, setores com baixa rotatividade de pacientes, visto que isso favoreceria o serviço farmacêutico de seguimento do paciente;

- Demandas: as farmacêuticas residentes indicaram os setores onde se documentava um maior número de problemas farmacoterapêuticos.

Após a observação participante as farmacêuticas residentes debateram sobre cada um dos pontos e elaboraram, em consenso, um diagnóstico para cada cenário.

\subsubsection{Etapa 2 - Preparação}


Esta etapa contemplou a Fundamentação teórica (julho 2016 a agosto de 2016) e o Planejamento estratégico do serviço (setembro 2016 a maio de 2017):

Fundamentação teórica: baseado no diagnóstico situacional, as farmacêuticas residentes sugeriram a clínica e o serviço a ser implantado diante da realidade do hospital. Posteriormente, uma busca sistemática foi realizada nos bancos de dados Scielo, Pubmed, Scopus. Esta busca foi complementada com uma pesquisa na Revista Brasileira de Farmácia Hospitalar e Serviços de Saúde a fim de compreender quais as possibilidades e viabilidades do serviço sugerido ser implantado.

Planejamento estratégico do Serviço: Diante do modelo (PDSA) Plan, Do, Act, Study foi identificado na literatura possíveis estratégias a serem utilizadas na implementação [15].

Nesta etapa foram construídos os processos de trabalho. A partir da escolha do setor mais apropriado, foi definido o fluxo de atendimento do serviço clínico, bem como os instrumentos a serem utilizados. Os fluxos de trabalho foram definidos sob a supervisão das Tutoras da Residência, e, posteriormente, apresentados aos profissionais do setor. Após o estabelecimento do fluxo, foi possível identificar na literatura as variáveis que implicam de alguma forma na assistência, conduzindo uma melhoria na condição clínica do paciente.

Posteriormente, os fluxos de trabalho foram aplicados e testados no setor para verificar a necessidade de adequações. Foram reportadas as mudanças necessárias e analisados os resultados prévios. Com base nos resultados, ajustes foram propostos, bem como foram organizados quanto às potencialidades e fragilidades, conforme Curatolo et al. (2015) [16].

\subsubsection{Etapa 3 - Implementação Inicial: Avaliação dos Resultados Preliminares da Implementação}

As conciliações foram realizadas conforme as etapas de coleta (melhor histórico possível do uso de medicamentos), checagem (verificação de discrepâncias entre a coleta e a prescrição do momento) e comunicação (com a equipe) [17]. Os dados foram coletados de setembro de 2017 a novembro de 2017, a partir da documentação das entrevistas do Serviço de Conciliação com o paciente e/ou o acompanhante atendidos neste período, bem como, por meio da análise das prescrições hospitalares documentadas nos prontuários. A comunicação com a equipe de saúde era realizada de forma verbal e escrita; a verbal de forma presencial no momento da visita médica e/ou com a enfermeira responsável pelo paciente no dia, e a escrita via evolução farmacêutica e ficha de conciliação anexa em prontuário. A tabulação das variáveis coletadas foi realizada em planilha Excel®, sendo elas: idade, sexo, comorbidades, medicamentos utilizados, necessidade de intervenção farmacêutica e alteração de prescrição após intervenção farmacêutica.

Para a avaliação dos resultados gerados pela implementação do Serviço de Conciliação de Medicamentos foram incluídos os prontuários dos indivíduos admitidos no setor de Clínica Médica, no período de setembro a novembro de 2017. Neste estudo, não foram registradas de forma categorizadas as discrepâncias e suas intencionalidades. Ademais, foram excluídos da pesquisa os prontuários dos pacientes que não foram entrevistados em um período de 48 horas após admissão. As variáveis coletadas foram apresentadas por meio de estatística descritiva em valores absolutos e porcentagens.

\subsection{Aspectos Éticos}

O trabalho foi aprovado pelo Comitê de Ética e Pesquisa da Universidade Federal de Sergipe sob o parecer $n^{\circ}$ 2489455 CAAE: 82361917.6.0000.5546.

\section{RESULTADOS}




\subsection{Descrição da implementação}

\subsubsection{Etapa 1 - Exploração}

O diagnóstico situacional foi realizado com quatro farmacêuticos e demonstrou que a Clínica Médica foi o setor mais propício para a implementação dos serviços clínicos, considerando aspectos como: menor rotatividade da equipe, média complexidade dos pacientes e maior tempo de permanência para possibilitar que a rotina da residência conseguisse se adequar a clínica (Tabela 1).

Diante de um setor com perfil de internamento de idosos, polimedicados e com tempo de internação superior a 5 dias, evidenciou-se a necessidade de iniciar os serviços clínicos providos por farmacêuticos pela Conciliação de Medicamentos, a fim de possibilitar a continuidade de tratamentos domiciliares, evitar eventos adversos e, posteriormente, implantar o seguimento farmacoterapêutico.

Tabela 1 - Diagnóstico situacional para a seleção do setor para implementação dos serviços clínicos providos por farmacêuticos em um Hospital de Ensino do Estado de Sergipe (2016-2017).

\begin{tabular}{|c|c|c|c|c|c|c|}
\hline & Infraestrutura & $\begin{array}{c}\text { Recursos } \\
\text { humanos }\end{array}$ & $\begin{array}{c}\text { Processos } \\
\text { de trabalho }\end{array}$ & Demandas & Infraestrutura & $\begin{array}{c}\text { Recursos } \\
\text { humanos }\end{array}$ \\
\cline { 2 - 7 } & $\begin{array}{c}\text { Setor que não } \\
\text { necessita de } \\
\text { intervenções } \\
\text { estruturais para } \\
\text { implementação do } \\
\text { serviço }\end{array}$ & $\begin{array}{c}\text { Profissionais com } \\
\text { abertura ao } \\
\text { trabalho } \\
\text { multiprofissional- } \\
\text { colaborativo }\end{array}$ & $\begin{array}{c}\text { Setores } \\
\text { com baixa } \\
\text { rotatividade } \\
\text { profissional }\end{array}$ & $\begin{array}{c}\text { Setor com } \\
\text { processo } \\
\text { de } \\
\text { trabalho } \\
\text { bem } \\
\text { delineados }\end{array}$ & $\begin{array}{c}\text { Setor que não } \\
\text { necessita de } \\
\text { intervenções } \\
\text { estruturais para } \\
\text { implementação } \\
\text { do serviço }\end{array}$ & $\begin{array}{c}\text { Profissionais com } \\
\text { abertura ao } \\
\text { trabalho } \\
\text { multiprofissional } \\
\text { colaborativo }\end{array}$ \\
\hline $\begin{array}{c}\text { Clínica } \\
\text { Médica }\end{array}$ & $\mathbf{X}$ & $\mathbf{X}$ & $\mathbf{X}$ & $\mathbf{X}$ & $\mathbf{X}$ \\
\hline Pediatria & & $\mathbf{X}$ & $\mathbf{X}$ & $\mathbf{X}$ & & $\mathbf{X}$ \\
\hline Clínica & & & $\mathbf{X}$ & & $\mathbf{X}$ \\
Cirúrgica & & & & & \\
\hline UTI & & & & & $\mathbf{X}$ \\
\hline
\end{tabular}

\subsubsection{Etapa 2 - Preparação}

Após a sugestão de serviço trazida na fase de exploração, foi realizada uma busca na literatura utilizando a seguinte estratégia: (hospital) AND (implementation) AND (medication reconciliation). Esta busca gerou 269 artigos, sendo 132 oriundos do SCOPUS, 135 do Pubmed e dois do Scielo. Além disso, foram identificados quatro artigos na Revista SBRAFH. Após a leitura dos títulos e resumos, foram identificados artigos que relatavam o processo de implementação da conciliação de medicamentos e estes foram lidos na íntegra. Os processos desenvolvidos e os indicadores de resultados foram destacados para fundamentar as etapas posteriores.

A partir do diagnóstico situacional e com o subsídio da fundamentação teórica, foram identificadas na literatura possíveis estratégias a serem utilizadas na implementação, tais como:

- Abordagem em fases da Implementação - consolidar em poucos setores antes de ampliar o serviço aumenta a chance de sustentabilidade [18].

- Uso do PDSA como estratégia de planejamento estratégico em serviços de conciliação $[15,16$, 19].

- Treinamento da equipe a fim de padronizar os processos [20, 21].

- Divulgação das estratégias de implementação - tanto os sucessos quanto as lições aprendidas com os esforços malsucedidos [18].

A identificação de instrumentos se deu via fundamentação teórica [22, 23]. Somado a isso, foi realizada a análise da viabilidade de uso de um formulário já existente em outro Hospital de Ensino do mesmo estado também gerido pela EBSERH (Apêndice A). Por conveniência, o formulário foi 
adotado visto que coletava as variáveis recomendadas na literatura e facilitava a comparação futura dos dados em ambos hospitais.

Para a elaboração do fluxo do Serviço Clínico de Conciliação de Medicamentos (Figura 2), foram realizados durante as tutorias da residência (15 sessões, média de 4h cada sessão) os debates sobre as etapas recomendadas na literatura, o treinamento dos profissionais e simulação do preenchimento dos instrumentos [1, 5], bem como aplicações do fluxo no setor para verificar a aplicabilidade do serviço. Desta forma, as etapas foram adequadas de acordo com a realidade do setor e seguiram o seguinte fluxo:

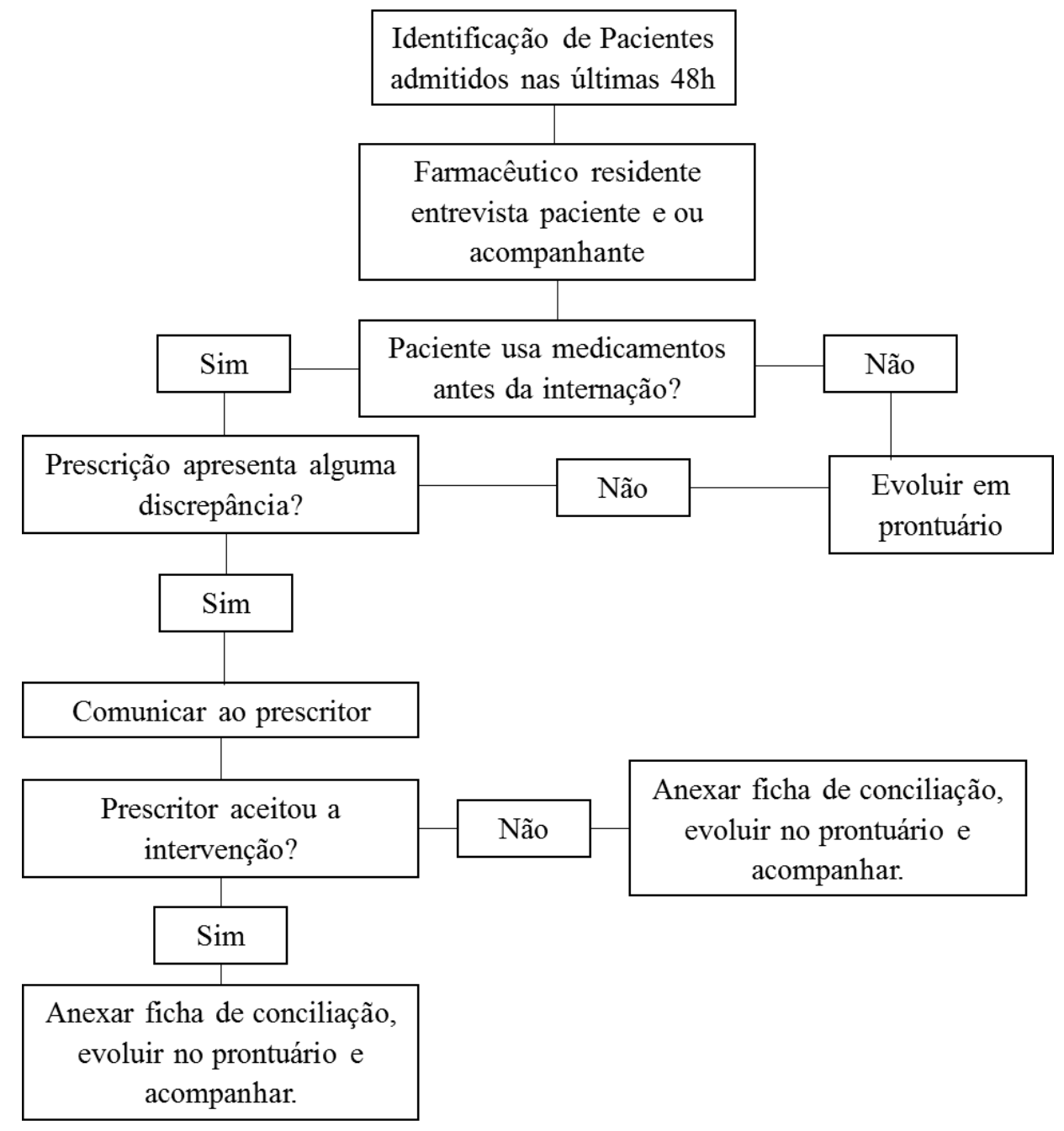

Figura 2:Fluxograma do Serviço de Conciliação de Medicamentos em um Hospital de Ensino do Estado de Sergipe (2016-2017).

- Cada paciente internado no setor foi entrevistado pelas farmacêuticas residentes, num período de, no máximo, 48 horas após admissão. A entrevista era realizada com o paciente, preferencialmente, mas em caso de desorientação ou dúvidas, o acompanhante foi para a obtenção da lista de medicamentos utilizados antes da internação.

- Foram coletadas informações sobre os medicamentos em uso pelo paciente antes da admissão hospitalar, como nome dos medicamentos, apresentação, dose, frequência e via de administração, assim como alergias e reações adversas previamente apresentadas pelo paciente.

- Todos os atendimentos realizados foram registrados em prontuário. Dados privativos do paciente (nome, número de prontuário ou endereço) não foram considerados e as demais informações dos prontuários foram acessadas conforme necessidade de consulta da prescrição. 
- A relação de medicamentos coletada foi comparada à prescrição hospitalar do paciente no momento da internação.

○ Em casos de discrepâncias, como omissão de algum medicamento, dose divergente da usual, duplicidade terapêutica ou interação medicamentosa, foi registrado em prontuário e realizado contato verbal com o médico responsável pela prescrição, para possível discussão do caso e avaliação clínica da conduta adotada.

- Na presença de discrepâncias, após evolução em prontuário, o paciente teve a ficha de conciliação anexa a sua última prescrição e acompanhamento de alterações na prescrição (compatíveis com o alerta sinalizado pelo farmacêutico).

- Independentemente da quantidade de medicamentos conciliados, consideramos uma intervenção farmacêutica por paciente. Para cada intervenção farmacêutica proposta, foi registrada a alteração ou não da prescrição.

- Diante da ausência de retorno médico por escrito em prontuário quanto a discrepância sinalizada pelo farmacêutico, a verificação do aceite das intervenções farmacêuticas foi observada a partir da modificação da prescrição. Assim, padronizou-se como acatadas as alterações realizadas em até 48 horas após intervenção registrada.

Por fim, foi realizada a identificação dos indicadores de resultados. Assim, variáveis como idade, sexo, comorbidades prevalentes, medicamentos utilizados, necessidade de intervenção e aceitabilidade das intervenções farmacêuticas foram recomendadas na literatura e consideradas neste estudo [24, 25].

\subsubsection{Etapa 3- Resultados preliminares da Implementação inicial}

Durante esta fase foi necessário adequar o fluxo de atendimento dos pacientes na Clínica Médica, inserir a evolução farmacêutica, bem como, anexar mais uma ficha ao prontuário. Nos casos de necessidade de intervenção farmacêutica, após identificação de discrepâncias entre a lista de medicamentos de uso prévio coletados em entrevista com paciente e a prescrição hospitalar atual, o prescritor era contactado verbalmente, em caso de ausência deste, o contato verbal foi realizado com a enfermeira plantonista. Após contato verbal com a equipe médica ou com as (os) enfermeiras (os), era descrita a discrepância em evolução farmacêutica na ficha multidisciplinar e anexada uma ficha ao prontuário com a descrição dos medicamentos e posologias utilizados previamente.

Durante três meses de estudo, 251 pacientes foram admitidos no setor selecionado, nos dias de atendimento dos residentes. Destes, 82 foram atendidos pelo serviço de Serviço de Conciliação de Medicamentos. Foram identificadas discrepâncias na conciliação de 41 (50,00\%) pacientes atendidos, sendo realizada intervenção farmacêutica para cada um destes pacientes. Dos outros 41 pacientes que não necessitaram de intervenção farmacêutica, 12 (14,63\%) não utilizavam medicamentos anterior à internação, nove $(10,98 \%)$ não sabiam informar quais medicamentos utilizavam antes de serem admitidos, sete $(8,54 \%)$ pacientes já tinham os medicamentos inseridos nas prescrições antes de serem atendidos pelo serviço de conciliação e $13(15,85 \%)$ pacientes não necessitaram de intervenção, mas não foi registrado os motivos do porquê não necessitaram de intervenção pela equipe.

Dos pacientes que necessitaram de intervenções 21 (51,22\%) eram do sexo feminino e 20 (48,78\%) do sexo masculino, adultos (idade média de 69,08 anos $\pm 13,6$ anos), admitidos por diversas condições clínicas. As comorbidades mais prevalentes foram relativas ao sistema cardiovascular e ao sistema endócrino: hipertensão $32(78,05 \%)$ e diabetes mellitus 20 (48,78\%). Os medicamentos mais utilizados pelos pacientes antes da internação foram classificados de acordo com o sistema de classificação Anatômica Terapêutica Química (Anatomical Therapeutic Chemical - ATC), e o grupo com maior prevalência foi o sistema cardiovascular $34(82,92 \%)$, seguido do grupo do trato alimentar e metabólico (43,90\%), sangue e órgãos hematopoiéticos $(29,27 \%)$ e sistema nervoso $(29,27 \%)$.

Durante a coleta do histórico de medicamentos, verificou-se uma média de 4,5 medicamentos em uso, por paciente, antes da admissão. Quanto à modificação das prescrições após as intervenções farmacêuticas, os prontuários foram analisados em média 48 horas após as intervenções farmacêuticas, a fim de avaliar quantas alterações foram realizadas e/ou evoluídas pelos prescritores a partir da sinalização do farmacêutico. Identificamos sete $(17,07 \%)$ prescrições alteradas totalmente compatíveis com as intervenções farmacêuticas, cinco $(12,20 \%)$ prescrições alteradas parcialmente compatíveis com as intervenções farmacêuticas e $29(70,73 \%)$ prescrições não tiveram alterações após a 
intervenção farmacêutica. Ademais, do total de pacientes que necessitaram de intervenções $(n=41), 28$ $(68,30 \%)$ já estavam sob posse dos medicamentos que utilizavam antes da internação no setor.

\subsubsection{Etapa 4: Implementação total}

A implementação total de um serviço pode ser assumida quando este se torna integrado e materializado nos procedimentos práticos, no fluxograma organizacional e nas políticas institucionais. Neste ponto, o serviço implementado torna-se totalmente operacional e fluído, as decisões são incorporadas pela equipe e são realizadas com o apoio dos gerentes e administradores, bem como, nesta etapa já há o reconhecimento social pelos pacientes do serviço executado [26].

Diante disso, conforme descrito em metodologia, a implementação deste serviço pode levar de dois a quatro anos para atingir o impacto total. Deste modo, essa etapa não será apresentada neste estudo, pois, os resultados apresentados ainda são considerados preliminares.

\subsubsection{Lições aprendidas em cada etapa de Implementação}

Embora todo processo de implementação tenha sido planejado previamente e seguindo referenciais teóricos [11], ainda assim, durante sua execução, observou-se ruídos na execução das etapas e as principais reflexões acerca de fortalezas e fragilidades estão descritos na Tabela 2.

Tabela 1: Fortalezas, fragilidades e lições aprendidas durante as etapas de Implementação do Serviço de Conciliação de Medicamentos em um Hospital de Ensino do Estado de Sergipe (2016-2017).

\begin{tabular}{|c|c|c|c|}
\hline & Etapa 1 & Etapa 2 & Etapa 3 \\
\hline Fortalezas & $\begin{array}{l}\text { Sistema estruturado de } \\
\text { busca na literatura }\end{array}$ & $\begin{array}{l}\text { Espaço reservado dentro } \\
\text { do organograma da } \\
\text { residência para planejar } \\
\text { esta fase (tutorias) }\end{array}$ & $\begin{array}{c}\text { Geração de dados } \\
\text { preliminares sobre o serviço }\end{array}$ \\
\hline Fragilidades & $\begin{array}{c}\text { Por conveniência foi } \\
\text { utilizada ficha de } \\
\text { conciliação de outra } \\
\text { instituição, embora a } \\
\text { mesma não atendesse todas } \\
\text { as recomendações da } \\
\text { literatura }\end{array}$ & $\begin{array}{l}\text { Não ter envolvido os } \\
\text { profissionais do setor e os } \\
\text { médicos nesta etapa, } \\
\text { sendo a discussão restrita } \\
\text { a equipe de farmacêuticos }\end{array}$ & $\begin{array}{c}\text { Não caracterização das } \\
\text { intervenções, das } \\
\text { discrepâncias e da aceitação } \\
\text { das intervenções pelos } \\
\text { médicos }\end{array}$ \\
\hline Lições & $\begin{array}{c}\text { Fazer adaptação do } \\
\text { material para torná-lo mais } \\
\text { completo, ou utilizar } \\
\text { instrumentos validados= }\end{array}$ & $\begin{array}{c}\text { Ampliar o trabalho } \\
\text { colaborativo desde a } \\
\text { concepção da implantação } \\
\text { de novos serviços clínicos } \\
\text { providos por } \\
\text { farmacêuticos }\end{array}$ & $\begin{array}{c}\text { Na implementação total } \\
\text { documentar em ficha de } \\
\text { conciliação: caracterização } \\
\text { das intervenções, das } \\
\text { discrepâncias e da aceitação } \\
\text { das intervenções pelos } \\
\text { médicos }\end{array}$ \\
\hline
\end{tabular}

Ademais, outras barreiras foram enfrentadas, como: ausência de experiência dos residentes em implementação de novos serviços, número escasso de profissional farmacêutico no hospital, inexistência de prontuário eletrônico, dificuldade do contato verbal com os médicos diaristas, que não tinham rotina de horários de atendimentos estabelecidos. Outro desafio é o serviço clínico ser exclusivamente realizado pelas farmacêuticas residentes, assim, cada semestre apenas umas das residentes realizava as conciliações na clínica, sem disponibilidade de dedicação exclusiva a essa atividade, limitando o tempo disponibilizado para essa atividade e o número de atendimentos.

Em contrapartida, alguns facilitadores podem ser apontados como agentes exitosos no processo de implementação da conciliação, como: inserção do farmacêutico na equipe multidisciplinar, sendo 
requisitado em discussões e demandas clínicas; apresentação prévia dos serviços clínicos providos por farmacêuticos para a equipe multidisciplinar e pacientes; fortalecimento das atividades farmacêuticas no ambiente clínico hospitalar.

\section{DISCUSSÃO}

Em 2009, a Society of Hospital Medicine propôs a realização de uma conferência sobre conciliação de medicamentos para a identificação de barreiras, oportunidades, papel das parcerias entre os diversos locais de cuidado, indicadores de processos e seu impacto na prevenção de danos aos pacientes. Como resultado, os participantes identificaram 10 recomendações-chave, a fim de promover a conciliação de medicamentos como uma estratégia de segurança ao paciente [18].

Embora após uma década, algumas recomendações ainda são pouco relatadas na literatura, especialmente a divulgação de resultados de processo de implementação da conciliação de medicamentos [27]. Isto também se deve a falta da melhoria da qualidade nas descrições das intervenções farmacêuticas, gerando um problema na mensuração dos resultados, pois com insuficiência de detalhes importantes, é difícil para os leitores e pesquisadores determinar o que foi realmente implementado [28].

Diante da inexistência de serviços clínicos providos farmacêuticos no hospital em estudo, as etapas de implementação foram realizadas gradualmente e foram delineadas com base nos indicadores de qualidade de serviços de saúde [8, 14], a fim de proporcionar uma estruturação inicial adequada ao serviço. A etapa de exploração, caracterizada pelo diagnóstico situacional, pode ser considerada como alicerce de todo o processo, visto que é uma ferramenta que permite conhecer como é a organização dos serviços de saúde [16].

Rocha et al. (2016) [29] destacaram que o diagnóstico situacional associado a ferramentas de qualidade foi essencial para a construção de um Programa de Segurança do Paciente em um hospital público de ensino. Caso esta etapa fosse negligenciada, e apenas as publicações na literatura fossem observadas [24, 25], provavelmente outro setor do hospital teria sido priorizado na implementação.

Dentro do ambiente hospitalar parte considerável dos problemas farmacoterapêuticos são oriundos dos erros de medicação [3, 30, 31]. Estima-se que durante uma entrevista com o paciente é possível identificar $70 \%$ destes problemas. Semelhante ao observado neste estudo, a literatura aponta que os problemas farmacoterapêuticos estão comumente relacionados à pacientes idosos, devido a quantidade de medicamentos utilizados [1]. Além disso, diversas vezes, essa vulnerabilidade é atribuída à comunicação ineficiente entre as diferentes equipes assistenciais e à perda de informações importantes durante seu trajeto dentro do hospital, pode prejudicar a terapia medicamentosa durante a internação hospitalar, resultando em eventos adversos [32, 33].

Assim, a seleção da conciliação de medicamentos como estratégia de resolução de tais problemas neste estudo segue as recomendações das principais organizações internacionais de promoção de segurança dos pacientes, por exemplo, Joint Commission, World Health Organization, National Institute for Clinical Excellence, National Patient Safety Agency e Institute for Healthcare Improvement. Para estas organizações a conciliação é considerada "padrão-ouro" para resolução de erros de medicação, assim, incentivam a sua implementação nas instituições de saúde em todos os níveis de cuidado [34]. No cenário nacional a segurança do paciente ganha mais notoriedade através da Portaria $\mathrm{N}^{\circ}$ 529, DE $1^{\circ}$ de abril de 2013 que institui o Programa Nacional de Segurança do Paciente (PNSP) e com a RDC No 36, de 25 de julho de 2013 que institui ações para a segurança do paciente em serviços de saúde através de metas que abordam a segurança na prescrição, administração, uso de medicamentos e comunicação segura $[35,36]$.

A etapa de preparação teve como objetivo preparar os residentes e os farmacêuticos para a implementação do serviço, utilizando das seguintes estratégias: avaliação inicial das barreiras e dos facilitadores para a implementação, além do treinamento dos profissionais e simulação do preenchimento dos instrumentos, bem como aplicações do fluxo no setor para verificar a aplicabilidade do serviço, organização pessoal e comunicação entre a equipe a fim de promover uma sensibilização do serviço implantado [16, 18]. 
Com relação aos resultados preliminares da implementação inicial, os grupos de medicamentos com maior prevalência dentre as discrepâncias foram semelhantes aos encontrados em outros estudos, tendo em vista que em ambos há prevalência de idosos [37, 38, 39].

Neste estudo, não foram registradas de forma categorizada as discrepâncias e suas intencionalidades, no entanto, entre os dados coletados em entrevista com o paciente e na prescrição hospitalar atual houve registro da intervenção farmacêutica e acompanhamento de seus desdobramentos pelas farmacêuticas residentes. A ausência desta categorização implica em todas as discrepâncias serem consideradas como erros de medicação, entretanto, sabe-se que discrepâncias intencionais, desde que documentadas, podem ser clinicamente relevantes [38 - 40]. Neste contexto, como os formulários utilizados neste estudo não contemplaram as categorizações e intencionalidades, ao final do trabalho o formulário foi adaptado, a fim de ter dados mais completos e fidedignos.

Uma das principais limitações encontradas neste estudo foi o número de farmacêuticos disponíveis para a conciliação, visto que a implementação do serviço envolveu apenas as farmacêuticas residentes. De acordo com os parâmetros mínimos para recursos humanos da SBRAFH, para o funcionamento da farmácia hospitalar, o recomendado seria um farmacêutico clínico em período integral por unidade clínica, sendo responsável por no máximo 30 leitos [41]. Assim, diante da impossibilidade de as farmacêuticas residentes estarem no setor em período integral, a comunicação com a equipe, seja verbal ou escrita, foi fragilizada, podendo ter repercutido inclusive nos dados de aceitação das intervenções. A inserção das residências multiprofissionais em saúde nos hospitais de ensino foi designada como uma estratégia de formação prática de recursos humanos qualificados, para o Sistema Único de Saúde. Contudo, além de preparar o profissional farmacêutico para os desafios de sua futura atuação, agrega oportunidades de implementação ou ampliação dos serviços farmacêuticos em hospitais de ensino $[42,43]$.

Um dos fatores que merece destaque, foram as barreiras enfrentadas durante as intervenções. A baixa adesão da equipe médica e consequentemente a não aceitação de muitas intervenções no processo de conciliação de medicamentos é resultante de um serviço ainda em construção, o qual necessita de mais profissionais Farmacêuticos que sejam atuantes na clínica contribuindo e fazendo parte da equipe prestadora do cuidado à saúde. A literatura demonstra que a inserção do farmacêutico clínico nas unidades promove redução do risco de complicações clínicas, diminuição de reinternamentos, tempo de internação e custos com medicamentos [44, 45]. Neste contexto, embora numericamente insuficiente para atender as demandas farmacoterapêuticas, a inserção das farmacêuticas residentes nas unidades, tornou-se um fator determinante para a qualidade na assistência e segurança do uso de medicamentos neste estudo. Deste modo, só foi possível a implementação da conciliação a partir do advento da residência.

\section{CONCLUSÃO}

Diante do fluxo apresentado, caso outros setores do hospital almejem a implementação da conciliação de medicamentos, este estudo poderá auxiliar na projeção dos processos nas etapas de exploração, preparação e implementação inicial. Além disso, este estudo revelou que metade dos pacientes atendidos necessitou de alguma intervenção farmacêutica, ressaltando a necessidade da atuação do farmacêutico nas unidades clínicas, minimizando potenciais erros de medicação, podendo atenuar interrupções no tratamento de condições crônicas que podem aumentar a possibilidade de agravo das comorbidades não tratadas. Ademais, a comunicação escrita não foi eficaz e a carência de ações colaborativas foram fatores que limitaram a aceitação das intervenções farmacêuticas.

Pesquisas futuras podem expandir o trabalho para outros setores do hospital, analisar detalhadamente as discrepâncias encontradas, estimar a economia gerada ao realizar a conciliação de medicamentos (farmacoeconomia), instruir e avaliar a percepção da equipe médica quanto aos serviços de conciliação de medicamentos.

\section{AGRADECIMENTOS}

Aos Farmacêuticos Residentes que colaboraram com esta pesquisa: Ailka Alinne Soares Tavares Silva, Fernanda Oliveira Prado, Joan Santos Cruz. A todos que compõe o programa de Residência 
Multiprofissional em Atenção Hospitalar à Saúde, a equipe da Farmácia Hospitalar do Hospital Universitário de Lagarto. Por fim, gratidão aos pacientes, familiares e cuidadores que forneceram os dados aqui apresentados.

\section{REFERÊNCIAS BIBLIOGRÁFICAS}

1. Conselho Federal de Farmácia (CFF). Serviços Farmacêuticos diretamente destinados ao paciente, à família e à comunidade: contextualização e arcabouço conceitual/Conselho Federal de Farmácia. Brasília: Conselho Federal de Farmácia, 2016. 84 p.

2. Donaldson LJ, Kelley ET, Dhingra-Kumar N, Kieny MP, Sheikh A. Medication Without Harm: WHO's Third Global Patient Safety Challenge. Lancet. 2017;389(10080):1680-81, doi:10.1016/S01406736(17)31047-4.

3. Hojat M, Gonnella J. An instrument for measuring pharmacist and physician attitudes towards collaboration: Preliminary psychometric data. J Interprof Care. 2010:25:66-72.

4. Vries de EN, Ramrattan MA, Smorenburg SM, Gouma DJ, Boermeester MA. The incidence and nature of inhospital adverse events: a systematic review. Qual Saf Health Care. 2008;17(3):216-223, doi:10.1136/qshc.2007.023622.

5. Schuch AZ, Zuckerman J, Santos MEF, et al. Reconciliação de medicamentos na admissão em uma unidade de oncologia pediátrica. Rev Bras Farm Hosp Serv Saúde. 2013;4:35-9.

6. World Health Organization. The High5s Project - Standard Operating Protocol for Medication Reconciliation. Disponível em: <https://www.who.int/patientsafety/implementation/solutions/high5s/h5ssop.pdf>. Acesso em 08 Dez. 2018.

7. Bemt PM, Schrieck-De Loos EM, Linden C, et al. Effect of medication reconciliation on unintentional medication discrepancies in acute hospital admissions of elderly adults: a multicenter study. J Am Geriatr Soc. 2013:61:1262-1268.

8. Mekonnen AB, McLachlan AJ, Brien JE. Effectiveness of pharmacist-led medication reconciliation programmes on clinical outcomes at hospital transitions: a systematic review and meta-analysis. BMJ Open. 2016;6(2): e010003.

9. Kaboli PJ, Hoth AB, McClimon BJ, Schnipper JL. Clinical pharmacists and inpatient medical care: a systematic review. Arch Intern Med. 2006 May;166(9):955-64.

10. Fernandes O. Medication Reconciliation in the Hospital What, why, where, when, who and how? Healthc Q. 2012;15:42-9.

11. Nilsen P. Making sense of implementation theories, models and frameworks. Implement Sci. 2015;10:53, doi:10.1186/s13012-015-0242-0.

12. Donabedian A. The quality of care. How can it be assessed? JAMA. 1988;260(12):1743-8, doi:10.1001/jama.260.12.1743.

13. Portela MC. Avaliação da qualidade em saúde. In: Rozenfeld S. Fundamentos da Vigilância Sanitária. Rio de Janeiro: Editora FIOCRUZ; 2000.

14. Moullin JC, Sabater-Hernández D, Benrimoj SI. Qualitative study on the implementation of professional pharmacy services in Australian community pharmacies using framework analysis. BMC Health Serv Res. 2016;16(1):439, doi:10.1186/s12913-016-1689-7

15. Deming WE. The new economics for industry, government, education. Cambridge: MIT Press; 2000. 265 p.

16. Curatolo N, Gutermann L, Devaquet N, et al. Reducing medication errors at admission: 3 cycles to implement, improve and sustain medication reconciliation. Int J Clin Pharm. 2015;37(1):113-20, doi: 10.1007/s11096-014-0047-2.

17. Silvestre CC, Lyra Jr DP. Os três C's da conciliação de medicamentos: realidade e perspectivas. Rev Bras Farm Hosp Serv Saúde 2018;9(1):e091.001, doi:10.30968/rbfhss.2018.091.001

18. Greenwald JL, Halasyamani LK, Greene J, et al. Making Inpatient Medication Reconciliation Patient Centered, Clinically Relevant, and Implementable: A Consensus Statement on Key Principles and Necessary First Steps. J Hosp Med. 2010;5(8):477-485, doi:10.1002/jhm.849

19. Lingaratnam S, Aranda S, Pearce T, Kirsa S. A controlled before and after study to evaluate a patient and health professional partnership model towards effective medication reconciliation. J Oncol Pharm Pract. 2013 Mar;19(1):48-56, doi: 10.1177/1078155212451936.

20. Sanchez SH, Sethi SS, Santos SL, Boockvar K. Implementing medication reconciliation from the planner's perspective: a qualitative study. BMC Health Serv Res. 2014;14:290, doi:10.1186/1472-6963-14-290

21. Freisinger A, Lám J, Barki L, et al. Feasibility of the implementation of medication reconciliation in Hungary. Orv Hetil. 2014;155(35):1395-1405, doi:10.1556/OH.2014.29976

22. The Joint Commission. Sentinel event data: Root causes by event type. 2014 [acesso em 04 out 2019]. Disponível em: http://www.jointcommission.org/sentinel_event_statistics/. 
23. Institute for Safe Medication Practices Canada. Medication Reconciliation (MedRec) [acesso em 04 out 2019]. Disponível em: https://www.ismp-canada.org/medrec/.

24. Hias J, Van der Linden L, Spriet I et al. Predictors for unintentional medication reconciliation discrepancies in preadmission medication: a systematic review. European Journal of Clinical Pharmacology. 2017;73(11):1355-77, doi: 10.1007/s00228-017-2308-1.

25. Spinewine A, Claeys C, Foulon V, et al. Approaches for improving continuity of care in medication management: a systematic review. Int J Quality Health Care. 2013;25(4):403-17, doi: 10.1093/intqhe/mzt032.

26. Fixsen DL et al. (2005). Implementation Research: A synthesis of the literature (FMHI \#231). Tampa, FL: University of South Florida, Louis de la Parte Florida Mental Health Institute, The National Implementation Research Network.

27. Brito GC. Clinical pharmacy services in units of Farmácia Popular do Brasil program in state of Sergipe: establishment, implementation and consolidation. 2015. 282 f. Tese (Doutorado em Ciências da Saúde) Universidade Federal de Sergipe, São Cristóvão, 2015.

28. Wilson PM, et al. Enhancing the reporting of implementation research. Implement Sci. 2017;12:13. doi: 10.1186/s13012-017-0546-3.

29. Rocha DJM, Wolf LDG, Haddad MCFL et al. Construção de programa de segurança do paciente em hospital público de ensino: pesquisa documental. Cogitare Enferm. 2016:21(4):01-07. doi: 10.5380/ce.v21i4.45604.

30. Frizon F, Santos AH, Caldeira LF, Menolli PVS. Reconciliação de medicamentos em hospital universitário. Rev enferm UERJ. 2014;22(4):454-60.

31. Nogueira JWS, Rodrigues MRCS. Comunicação efetiva no trabalho em equipe em saúde: um desafio para a segurança do paciente. Cogitare Enferm. 2015;(3):630-4.

32. Lindenmeyer LP, Goulart VP, Hegele V. Reconciliação Medicamentosa como Estratégia para a Segurança do Paciente Oncológico: Resultados de um estudo piloto. Rev Bras Farm Hosp Serv Saúde. 2013;4(4):51-5.

33. Alves LR, Giacomini MA, Camelo SHH, et al. Evidências sobre trabalho em equipe na atenção hospitalar / Evidence on teamwork in hospital care / Evidencias sobre trabajo en equipo en la atención hospitalaria. J Health NPEPS. 2016;1(2):246-62.

34. Silvestre, CC. Conciliação de medicamentos: fatores de risco, documentação da prática e desenvolvimento de instrumento de avaliação - Aracaju, 2018. 149 f. Tese (doutorado em Ciências da Saúde) - Universidade Federal de Sergipe, 2018.

35. Brasil. Portaria $\mathrm{N}^{\mathrm{o}} 529$, de $1^{\mathrm{o}}$ de abril de 2013. Institui o Programa Nacional de Segurança do Paciente (PNSP). Ministério da Saúde, Brasília, 2013.

36. Brasil. Resolução $n^{\circ} 36$, de 25 de julho de 2013b. Institui ações para a segurança do paciente em serviços de saúde e dá outras providências. Diário Oficial da União, Brasília, 2013.

37. Lombardi NF, et al. Análise das discrepâncias encontradas durante a conciliação medicamentosa na admissão de pacientes em unidades de cardiologia: um estudo descritivo. Rev. Latino-Am. Enfermagem, 2016:24:1-7, doi: 10.1590/1518-8345.0820.2760 Disponível em: <http://www.scielo.br/scielo.php?pid=S010411692016000100383\&script=sci_arttext\&tlng=pt $>$ Acesso em 08 Dez.2017.

38. Silvestre CC. Necessidade Da Conciliação De Medicamentos: Avaliação Da História Da Farmacoterapia De Pacientes Admitidos Em Um Hospital Universitário. Sergipe. Tese [Mestrado em ciências da saúde] Universidade Federal de Sergipe; 2013. Disponível em: 〈http://ri.ufs.br/jspui/handle/riufs/3957>. Acesso em: 15 dez. 2017.

39. Mendes AEM. Conciliação de medicamentos na admissão hospitalar: um ensaio Clínico randomizado. Curitiba. Dissertação [Mestrado em Medicina Interna e Ciências da Saúde] - Universidade Federal do Paraná; 2016.

40. Soares AS. Inconsistências medicamentosas em hospital no sul do Brasil: a importância da reconciliação medicamentosa na segurança do paciente. Santa Catarina. Dissertação [Mestrado em Ciências da Saúde] Universidade Do Sul De Santa Catarina; 2016.

41. Sociedade Brasileira de Farmácia Hospitalar e Serviços de Saúde. Padrões mínimos para farmácia hospitalar e serviços de saúde. São Paulo; 2017.

42. Santos FS, Freitas EP, Costa JM. Associação entre ocorrência de reações adversas e realização de intervenções farmacêuticas em um hospital de ensino. Rev. Bras. Farm. Hosp. Serv. Saúde; 2016:7(2):8-12.

43. Heleodoro NM, Gonçalves RCR. A residência multiprofissional e a mão de um serviço de atenção farmacêutica [editorial]. Braz J Health Res. 2011;13(3):1-2.

44. Alcântara TS. Análise diagnóstica para implantação de serviços de farmácia clínica em um hospital público de alta complexidade. Aracaju. Dissertação [Pós-Graduação em Ciências as Saúde] - Universidade Federal de Sergipe; 2016.

45. Rodrigues JPV, Pereira LRL. Farmácia Clínica em Ambiente Hospitalar: Perspectivas e Estratégias para Implementação. J Appl Pharm Sci. 2016;7-10. 
